\title{
NONCOMMUTATIVE GEOMETRY AND GEOMETRIC PHASES
}

\author{
B. Basu $⿴ 囗 十$ and Subir Ghost \\ Physics and Applied Mathematics Unit \\ Indian Statistical Institute \\ Kolkata-700108 \\ S. Dhall \\ S. A. Jaipuria College \\ Kolkata- 700005
}

\begin{abstract}
We have studied particle motion in generalized forms of noncommutative phase space, that simulate monopole and other forms of Berry curvature, that can be identified as effective internal magnetic fields, in coordinate and momentum space. The Ahranov-Bohm effect has been considered in this form of phase space, with operatorial structures of noncommutativity. Physical significance of our results are also discussed.

PACS numbers: $14.80 . \mathrm{Hv}, 11.10 . \mathrm{Nx}, 03.65 .-\mathrm{w}$
\end{abstract}

\section{INTRODUCTION}

The possible existence of magnetic monopole (MM) was first discussed by Dirac [1] and later in [2] in non-abelian gauge theory.

However, recently [3] the signatures of MM in crystal momentum space in $\mathbf{S r R u O}_{\mathbf{3}}$, (a ferromagnetic crystal), have appeared as peaks in transverse conductivity $\sigma_{x y}$. The MM formation in low energy regime $(\sim 0.1-1 \mathrm{eV})$ in the condensed matter system [3], (as compared to the predicted range $\sim 10^{16} \mathrm{GeV}$ in particle physics [2]), is obviously the reason for their observation in the former. The MM in $\sigma_{x y}$ is again directly linked to Anomalous Hall Effect (AHE) where $\sigma_{x y}$ is identified as the Berry curvature. The very intrinsic origin of AHE [4], independent of external magnetic fields, suggests [5] that the whole phenomena might be interpreted as a motion of (Bloch) electrons in a non-trivial symplectic manifold with the symplectic two-form being essentially the Berry curvature. This is a specific form of NonCommutative (NC) space (see for example [ 6$]$ ), that appears because one introduces [3, 7] a gauge covariant position operator $x_{\mu} \equiv i \partial_{\mu}-a_{n \mu}(\vec{k}), a_{n \mu}=i<u_{n \vec{k}}\left|\frac{\partial}{\partial k_{\mu}} u_{n \vec{k}}\right\rangle$, for the Bloch wavefunction $\psi_{n \vec{k}}(r)=\exp (i \vec{k} \cdot \vec{r}) u_{n \vec{k}}(r)$, with the coordinates satisfying the NC structure, $\left[x_{\mu}, x_{\nu}\right]=-i F_{\mu \nu}, \quad F_{\mu \nu}=$ $\partial_{k_{\mu}} a_{n \nu}-\partial_{k_{\nu}} a_{n \mu}$. This NC induces the additional anomalous part of velocity that yields the AHE. Notice that for the crystalline system in question, Bloch states are the natural setting and the curvatures become functions of momenta as expected. Clearly $a_{n \mu}$ is unrelated to any external source and is generated within.

In a recent paper [8] real space Berry phase manifests as a further contribution in AHE in AuFe alloy and the underlying theory [9] requires a topologically non-trivial spin configuration. The theory 9] indicates that the coupling between this net spin chirality and a global magnetization, (which might be spontaneous, as in $\mathbf{A u F e}$ alloy), plays a crucial role. Onoda, Tatara and Nagaosa in [9] have argued that the real space and momentum space Berry phase manifest itself in two different regimes. The real space vortex is a good picture in disordered case (equivalently for short electrons with mean free path) whereas momentum space vortex is a useful model in the pure case [10]. A re-entrant ferromagnet, such as the AuFe alloy is a sample of the former kind. It should also be remembered that [10] in principle, complicated structures of Berry curvature are indeed possible depending on the particular nature of a sample, although so far the only numerical work concerns the simple monopole form, as observed in [3]. Besides, a study of the underlying geometry of the ferromagnetic spin system shows that the inherent

*Electronic address: banasri@isical.ac.in

$\dagger$ Electronic address: subir ghosh2@rediffmail.com

‡Electronic address: sarmi $30 @$ rediffmail.com 
magnetic type of behavior is caused by the Berry curvature in real space which arises due to the spin rotations of conducting electrons and is the effect of noncommutativity in momentum space [? ].

Keeping this background in mind, we put forward forms of NC space that can induce singular behavior (in the effective magnetic field) in coordinate space. Different novel structures of Berry curvature appear in our framework. Incidentally, our work is a generalization of the work of [5]. The NC structure and its associated symplectic form, considered in [5], was not general enough to allow the vortex structures that we have obtained here.

With this special form of $\mathrm{NC}$ space we have calculated the

Aharanov- Bohm (AB) phase and have shown that there is a modification term due to the noncommutativity of space-space coordinates. This leads to new expression and bound for $\theta$ - the noncommutativity parameter.

The paper is organized as follows: In Section II we introduce the particular form of NC space that will be studied subsequently. Section III deals with the Lagrangian formulation of the model and the related dynamics in a general way. Section IV is devoted to the study of the Aharanov-Bohm effect in this specific NC phase space. In Section $\mathbf{V}$ we discuss the physical implications of our findings.

\section{NONCOMMUTATIVE PHASE SPACE}

We start by positing a non-canonical phase space that has the Snyder form of spatial noncommutativity and at the same time the momenta satisfies a conventional monopole algebra. Similar structures have also appeared in [11]. In the beginning we have introduced two distinct $\mathrm{NC}$ parameters $\theta$ and $b$ for the above two independent forms of noncommutativity so that their individual roles can be observed.

The phase space is given below:

$$
\begin{aligned}
\left\{X_{i}, X_{j}\right\} & =-\theta\left(X_{i} P_{j}-X_{j} P_{i}\right), \\
\left\{X_{i}, P_{j}\right\} & =\delta_{i j}-\theta P_{i} P_{j}, \\
\left\{P_{i}, P_{j}\right\} & =b \epsilon_{i j k} \frac{X_{k}}{X^{3}}
\end{aligned}
$$

where $X=\sqrt{X^{2}}$.

We discuss rotational properties of the vectors. From the definition of the angular momentum, $L_{j}=$ $\epsilon_{j k l} X_{k} P_{l}$ we have the following commutation relations,

$$
\begin{aligned}
& \left\{X_{i}, L_{j}\right\}=\epsilon_{i j k} X_{k}, \\
& \left\{P_{i}, L_{j}\right\}=\epsilon_{i j k} P_{k}+b\left(\frac{X_{i} X_{j}}{X^{3}}-\delta_{i j} \frac{1}{X}\right), \\
& \left\{L_{i}, L_{j}\right\}=\epsilon_{i j k} L_{k}+b \epsilon_{i j k} \frac{X_{k}}{X} .
\end{aligned}
$$

Notice that $b$ (and not $\theta$ ) destroys the transformation properties. But this is expected since as is well known the Snyder algebra does not clash with rotational invariance. To restore the angular momentum algebra consider the term

$$
S_{j}=-d \frac{X_{j}}{X}
$$

which yields the total angular momentum as

$$
\tilde{L}_{j}=L_{j}+S_{j} .
$$

We naturally identify $\vec{S}$ as the effective spin vector, that is induced by the algebra (1). Now angular momentum algebra is given by

$$
\begin{aligned}
& \left\{X_{i}, \tilde{L}_{j}\right\}=\epsilon_{i j k} X_{k}-\theta d\left(-\frac{X_{i} P_{j}}{X}+\frac{X_{i} X_{j}(X . P)}{X^{3}}\right) \\
& \left\{P_{i}, \tilde{L}_{j}\right\}=\epsilon_{i j k} P_{k}+(b-d)\left(\frac{X_{i} X_{j}}{X^{3}}-\delta_{i j} \frac{1}{X}\right)-\theta d\left(\frac{P_{i} P_{j}}{X}-\frac{P_{i} X_{j}(X . P)}{X^{3}}\right) \\
& \left\{\tilde{L}_{i}, \tilde{L}_{j}\right\}=\epsilon_{i j k} \tilde{L}_{k}+(b-d) \epsilon_{i j k} \frac{X_{k}}{X}
\end{aligned}
$$


Putting $d=b$ the algebra is as follows

$$
\begin{aligned}
& \left\{X_{i}, \tilde{L}_{j}\right\}=\epsilon_{i j k} X_{k}-\theta b\left(-\frac{X_{i} P_{j}}{X}+\frac{X_{i} X_{j}(X . P)}{X^{3}}\right) \\
& \left\{P_{i}, \tilde{L}_{j}\right\}=\epsilon_{i j k} P_{k}-\theta b\left(\frac{P_{i} P_{j}}{X}-\frac{P_{i} X_{j}(X . P)}{X^{3}}\right) \\
& \left\{\tilde{L}_{i}, \tilde{L}_{j}\right\}=\epsilon_{i j k} \tilde{L}_{k}
\end{aligned}
$$

The above considerations prompt us to study a simpler NC algebra, with $b=\theta$,

$$
\begin{aligned}
\left\{X_{i}, X_{j}\right\} & =-\theta\left(X_{i} P_{j}-X_{j} P_{i}\right), \\
\left\{X_{i}, P_{j}\right\} & =\delta_{i j}-\theta P_{i} P_{j}, \\
\left\{P_{i}, P_{j}\right\} & =\theta \epsilon_{i j k} \frac{X_{k}}{X^{3}}
\end{aligned}
$$

Hence the NC algebra is governed by a single NC parameter $\theta$.

Hence to the approximation (i.e. $O(\theta)$ ) that we are interested in and ignoring the terms which are in order of $\theta^{2}$, we find,

$$
\begin{aligned}
& \left\{X_{i}, \tilde{L}_{j}\right\}=\epsilon_{i j k} X_{k}, \\
& \left\{P_{i}, \tilde{L}_{j}\right\}=\epsilon_{i j k} P_{k}, \\
& \left\{\tilde{L}_{i}, \tilde{L}_{j}\right\}=\epsilon_{i j k} \tilde{L}_{k} .
\end{aligned}
$$

Thus, to the lowest non-trivial order in $\theta$, is possible to define an angular momentum operator in a consistent way.

Since the form of noncommutativity is operatorial in nature we must check that the Jacobi identities are satisfied,

$$
J(A, B, C) \equiv\{A,\{B, C\}\}+\{B,\{C, A\}\}+\{C,\{A, B\}\}=0
$$

We obtain,

$$
J\left(X_{i}, X_{j}, X_{k}\right)=J\left(X_{i}, X_{j}, P_{k}\right)=J\left(X_{i}, P_{j}, P_{k}\right)=0 .
$$

The Jacobi identity between transformed angular momentum is also satisfied,

$$
J\left(\tilde{L}_{i}, \tilde{L}_{j}, \tilde{L}_{k}\right)=0 .
$$

However the Jacobi identity between momenta is violated,

$$
\begin{array}{r}
J\left(P_{i}, P_{j}, P_{k}\right)=-3 \theta \epsilon_{i j k} \frac{1}{X^{3}}+3 \theta \frac{X_{l}}{X^{5}}\left(\epsilon_{i j l} X_{k}+\epsilon_{j k l} X_{i}+\epsilon_{k i l} X_{j}\right) \\
=-\theta \epsilon_{i j l} \partial_{l}\left(\frac{X_{k}}{X^{3}}\right)-\theta \epsilon_{j k l} \partial_{l}\left(\frac{X_{i}}{X^{3}}\right)-\theta \epsilon_{k i l} \partial_{l}\left(\frac{X_{j}}{X^{3}}\right) .
\end{array}
$$

But from the analysis of Jackiw [1] we know the implications of this violation and how to live with it.

\section{SYMPLECTIC DYNAMICS}

Non-violation of the Jacobi identities (at least up to the prescribed order) is essential in our case since we wish to study the dynamics by exploiting the elegant scheme of Faddeev and Jackiw [12] and follow the notation of a recent related work [13].

A generic first order Lagrangian, expressed in the form,

$$
L=a_{\alpha}(\eta) \dot{\eta}^{\alpha}-H(\eta),
$$


where $\eta$ denotes phase space variables, leads to the Euler-Lagrange equations of motion,

$$
\omega_{\alpha \beta} \dot{\eta}^{\beta}=\partial_{\alpha} H, \omega_{\alpha \beta}=\partial_{\alpha} a_{\beta}-\partial_{\beta} a_{\alpha} .
$$

where $\omega_{\alpha \beta}$ denotes the symplectic two form. The inverse of the symplectic matrix is given by $\omega^{\alpha \beta}$.

$$
\omega^{\alpha \beta} \omega_{\beta \gamma}=\delta_{\gamma}^{\alpha}
$$

For our model, following (7), $\omega^{\alpha \beta}$ is defined by,

$$
\omega^{\alpha \beta}=\left(\begin{array}{cc}
-\theta\left(X_{i} P_{j}-X_{j} P_{i}\right) & \left(\delta_{i j}-\theta P_{i} P_{j}\right) \\
-\left(\delta_{i j}-\theta P_{i} P_{j}\right) & \theta \epsilon_{i j k} \frac{X_{k}}{X^{3}}
\end{array}\right) .
$$

The particle dynamics is easily derived in a straightforward way exploiting (15). We have,

$$
\dot{X}_{i}=\left\{X_{i}, H\right\} ; \quad \dot{P}_{i}=\left\{P_{i}, H\right\} .
$$

Considering a simple form of $H$,

$$
H=\frac{P^{2}}{2 m}+V(X)
$$

we obtain,

$$
\begin{gathered}
\dot{X}_{i}=\frac{1}{m}\left(1-\theta P^{2}\right) P_{i}+\theta\left((E . P) X_{i}-(E . X) P_{i}\right), \\
\dot{P}_{i}=E_{i}-\theta(E . P) P_{i}+\frac{\theta}{m} \epsilon_{i j k} P_{j} \frac{X_{k}}{X^{3}} .
\end{gathered}
$$

In the above we have identified $-(\partial V) /\left(\partial X_{i}\right) \equiv E_{i}$, the external electric field. We can rewrite the equations of motion as,

$$
\begin{gathered}
\dot{\vec{X}}=\frac{\vec{P}}{m^{*}}+\theta(\dot{\vec{P}} \times \vec{L}), \\
\dot{\vec{P}}=\vec{E}-\theta(\vec{E} \cdot \vec{P}) \vec{P}+\frac{\theta}{m X^{2}}(\vec{P} \times \vec{S}),
\end{gathered}
$$

where $m^{*}=m\left(1-\theta P^{2}\right)^{-1}$ and the spin vector $\vec{S}$ has been defined in (3). It is interesting to note that the origins of Berry curvature terms in (19) and (20) are different: the Snyder form of spatial noncommutativity in (11) is responsible for the former, whereas monopole form of the momentum noncommutativity in (11) for the latter. We can also express (19) as

$$
\dot{\vec{X}}=\frac{\vec{P}}{m^{*}}+\theta(\vec{E} \times \vec{L}) .
$$

It is straightforward to iterate (17) once again so that we obtain a generalized Lorentz force equation in the following form,

$$
\ddot{X}_{i}=\frac{1}{m}\left(1-\theta P^{2}\right) E_{i}-\frac{3 \theta}{m}(E . P) P_{i}+\frac{\theta}{m} \epsilon_{i j k} \frac{P_{j} X_{k}}{X^{3}}-\theta \epsilon_{i j k}(\vec{E} \times \vec{X})_{k} E_{j} .
$$

We will study the significance of these equations in the Discussion, Section V, at the end. 


\section{THE AHARONOV-BOHM EFFECT ON NC (SNYDER) SPACE}

In non-commutative space many interesting quantum mechanical problems have been studied extensively: such as hydrogen atom spectrum in an external magnetic field [14, 15], Aharonov-Bohm (AB) [16, 17], Aharonov-Casher effects [18], to name a few. However, all the above works have considered a constant form spatial noncommutativity. In the present work, for the first time, we consider such effects in the presence of an operatorial form of noncommutativity. Here we consider a purely Snyder form of noncommutative space,

$$
\begin{aligned}
\left\{X_{i}, X_{j}\right\} & =-\theta\left(X_{i} P_{j}-X_{j} P_{i}\right), \\
\left\{X_{i}, P_{j}\right\} & =\delta_{i j}-\theta P_{i} P_{j}, \\
\left\{P_{i}, P_{j}\right\} & =0
\end{aligned}
$$

that we obtained from (1) by putting $\left\{P_{i}, P_{j}\right\}=0$.

In the commutative Aharonov-Bohm effect, the presence of the flux produces a shift in the interference pattern. The value of the flux is such that the position of maxima and minima are interchanged due to a change of $\pi$ in the phase and vanishes when magnetic field is quantized. For noncommutative AharonovBohm effect a velocity dependent extra term in the flux arises even in the presence of quantized magnetic field [16]. This could be experimentally measured. The velocity can be so chosen that the phase shift become $2 \pi$ or integer multiple of $2 \pi$. So this phase shift might not be observed. The Aharonov-Bohm effect in noncommutative case can also be worked out using path integral formulation [16]. Electrons moving on a noncommutative plane in uniform external magnetic and electric field represents usual motion of electrons in an effective magnetic field. The related AB phase can be calculated and it yields the same effective magnetic field [17]. Using non-commutative quantum mechanics Aharonov-Bohm phase can be obtained on NC phase space [17].

For the NC phase space (23), the variables $X_{i}, P_{j}$ can be expressed in terms of canonical (Darboux) set of variables $x_{i}, p_{j}$ :

$$
X_{i}=x_{i}-\theta(x . p) p_{i} ; \quad P_{i}=p_{i}
$$

The $x_{i}, p_{j}$ satisfy

$$
\left\{x_{i}, p_{j}\right\}=\delta_{i j} ; \quad\left\{x_{i}, x_{j}\right\}=\left\{p_{i}, p_{j}\right\}=0 .
$$

Let $H(X, P)$ be the Hamiltonian operator of the usual quantum system, then the Schrödinger equation on $\mathrm{NC}$ space is written as

$$
H(X, P) * \psi=E \psi
$$

The star product can be changed into the ordinary product by replacing $H(X, P)$ with $H(x, p)[19]$. Thus the Schrödinger equation can be written as,

$$
H\left(X_{i}, P_{i}\right) \psi=H\left(x_{i}-\theta(x . p) p_{i} ; p_{i}\right) \psi=E \psi .
$$

When magnetic field is applied, the Schrödinger equation becomes

$$
H\left(X_{i}, P_{i}, A_{i}\right) * \psi=E \psi .
$$

Now we also need to replace the vector potential $A_{i}$ with a phase shift as given by

$$
\mathcal{A}_{i} \rightarrow A_{i}-\frac{1}{2} \theta(x \cdot p) p_{j} \partial_{j} A_{i} .
$$

So, the Schrödinger equation (27) in the presence of magnetic field becomes

$$
H\left(x_{i}-\theta(x . p) p_{i} ; p_{i} ; A_{i}-\frac{1}{2} \theta(x . p) p_{j} \partial_{j} A_{i}\right) \psi=E \psi .
$$

If $\psi_{0}$ is the solution of (29) when $A_{i}=0$, then the general solution of 291) may be given by

$$
\psi=\psi_{0} \exp \left[i q \int_{x_{0}}^{x}\left(A_{i}-\frac{1}{2} \theta(x . p) p_{j} \partial_{j} A_{i}\right) d x_{i}\right] .
$$


The phase term of (30) is called the AB phase. In a double slit experiment if we consider the charged particle of charge $q$ and mass $m$ to pass through one of the the slits, then the integral in (30) runs from the source $x_{0}$ to the screen $x$, the interference pattern will depend on the phase difference of two paths. The total phase shift for the $\mathrm{AB}$ effect is

$$
\left.\Delta \Phi_{A B}=\delta \Phi_{0}+\delta \Phi^{N C}=i q \oint A_{i} d x_{i}-i \frac{q}{2} \theta \oint\left(m v_{j}+q A_{j}\right)\left(m v_{k}+q A_{k}\right) x_{k} \partial_{j} A_{i}\right) d x_{i},
$$

where we use the equation $m v_{l}=p_{l}-q A_{l}$. The line integral runs from the source through one slit to the screen and returns to the source through another slit. The first term of (31) is the usual AB phase. One of the four $\theta$ dependent term in (31) is $\left.\oint v_{j} v_{k} x_{k} \partial_{j} A_{i} d x_{i}=\vec{\nabla} A_{i}\right)(\vec{v} \cdot \vec{x}) d x_{i}$. The rest are computed in a similar way.

Adding all the terms we can write the $\mathrm{AB}$ phase as

$$
\Delta \Phi_{A B}=i q \oint A_{i} d x_{i}-i \frac{q}{2} \theta \oint[(m \vec{v}+q \vec{A}) \cdot \vec{x}]\left[(m \vec{V}+q \vec{A}) \cdot \vec{\nabla} A_{i}\right] d x_{i}
$$

Previous results [16, 17] with a constant form of spatial noncommutativity are of the form,

$$
\Delta \Phi_{A B}^{N C} \sim i \frac{q}{2} \oint\left[\vec{\theta} .(m \vec{v}+q \vec{A}) \times \vec{\nabla} A_{i}\right] d x_{i} .
$$

Comparing our result with the above (33), we find that the $\mathrm{NC}$ correction in our case altogether a different structure and also there appears an extra piece $(m \vec{v}+q \vec{A}) \cdot \vec{x}$, which is a consequence of the form of space-space noncommutativity chosen in our model. We discuss the implications of our result in the Discussion section.

\section{DISCUSSIONS:}

Let us summarize and motivate our results. Our aim has been to demonstrate effective models of interest, (specially in the context of Condensed Matter physics), can be simulated compactly in a purely Hamiltonian formulation, developed in a suitable noncommutative space. The advantage is that one can have a simple form of Hamiltonian and the complicated responses of the system are induced by the noncommutative structure of spacetime. To be specific, in the present work in Section III, we have shown that effective spin-contributions are generated in our model, only from the symplectic structure with no explicit spin-term as such.

It was shown in [5] that the anomalous velocity term related to spin Hall effect has a natural interpretation in terms of a noncommutative space. We have shown that this result can be generalized in various possible ways: One can have Berry curvature terms both in coordinate as well as in momentum space and the singularity structure of the Berry potential, (not shown here), can be more complicated than that of a monopole. This will become apparent if one inverts $\omega^{\alpha \beta}$ in (15) to recover the Berry potential.

A novel form of anomalous velocity term has been derived in (17). From the equivalent form (19) we infer that there will be a deviation in the particle trajectory in the presence of an electric field [20].

On the other hand, in (20) we have an explicitly spin dependent term in the expression of $\dot{\vec{P}}$ with a coordinate space singularity. Once again, in the alternative force equation in (22), the leading term in low energy $\theta \frac{\vec{P} \times \vec{S}}{X^{2}}$ reminds us of models with the Rashba type of interactions [21]. Hence, these effects can be relevant for the studies in $[\underline{8}, 9]$.

Now we come to the results obtained in Section IV and their implications. As we have mentioned in Section IV, we consider the Snyder noncomutative space, as given in (23). As we have pointed out in Section IV, in the present case, the $\theta$-contribution in the AB phase, (derived for the constant noncommutative case [16, 17]), gets multiplied by a dynamical factor. This leads to some interesting consequences. As in previous cases [16], we can also derive a bound on $\theta$ pertaining to experimental observations. We compute $\gamma$, the ratio of the $\mathrm{AB}$ phases appearing in the normal case and noncommutative case:

$$
\gamma \equiv \frac{\Delta \phi^{N C}}{\Delta \phi} \sim\left(\frac{\theta}{R \lambda_{e}} \frac{v}{c}\right)\left(\frac{R}{\lambda_{e}} \frac{v}{c}\right) \approx \frac{\theta}{\lambda_{e}^{2}}\left(\frac{v}{c}\right)^{2}
$$


In (34), $\Delta \phi^{N C}$ corresponds to the $\theta$-contribution in (32) and $\Delta \phi$ refers to the $\theta=0$ commutative case, $R$ denotes the electron radius in the experimental setup and $\lambda_{e}$ is the Compton wavelength of the electron. Interestingly, in the present case, the extra dynamical factor cancels $R$ in $\gamma$ and reproduces the bound in the $R$-independent form:

$$
\sqrt{\theta}<\left(\frac{v}{c}\right)^{-1} \lambda_{e}
$$

This is distinct from the previously obtained expressions [16] but the bound is much lowered than that of 16 .

Finally, we would like to make a remark on the effect a generic noncommutative space can have in the study of inequivalent quantization in a non-simply connected manifold [22]. It is well known that $\mathrm{AB}$ effect is a prototype example of a multiply connected domain since the region of the solenoid that carries the magnetic flux is inaccessible to the charged particle. This leads to a punctured manifold $Q=\mathbb{R}^{2}-\delta$, ( $\delta$ denoting the solenoidal area), with a non-trivial first homotopy group $\Pi_{1}(Q)=Z$. One can still work in the trivial homotopy sector, but this requires additional topological terms in the action. They clearly show up in the path-integral quantization of the system. These issues have been extensively studied in [22], for the normal (commutative) spacetime. As it has been established here and before 16, 17], that noncommutative nature of spacetime generates additional contributions in the AB phase, clearly this will directly affect the above mentioned quantization programme. From the study of the modified quantization conditions, it might be possible to set an independent bound on $\theta$. We intend to study this aspect in future.

Acknowledgements: We would like to thank Prof.P.Horvathy for discussions and Prof.G.Tatara and Prf.D.Xiao for correspondences. Also we thank the Referees for the constructive comments.

[1] P.A.M.Dirac, Proc.Roy.Soc.London 133(1931)60 ;

R.Jackiw, Phys.Rev.Lett. 54(1985)159; For a different perspective and more related to our work, see R.Jackiw, Int.J.Mod.Phys. A19S1(2004)137.

[2] G. t'Hooft, Nucl.Phys. B79 (1974) 276; A.M.Polyakov, JETP Lett. 20 (1974)194.

[3] M.Onoda and N.Nagaosa, J.Phys.Soc.Jpn. 71(2002) 19; Z.Fang et al., Science 302 (2003) 92.

[4] R.Karplus and J.M.Luttinger, Phys.Rev. 95(1954)1154.

[5] A.Berard and H.Mohrbach. Phys.Rev. D69 95 (2004) 127701.

[6] H. S. Snyder, Phys. Rev. 71(1947) 38, N.Seiberg and E.Witten, JHEP 9909(1999)032, R.J.Szabo, Phy. Rep. 378 (2003)207, J. Lukierski, H. Ruegg, W.J. Zakrzewski, Annals Phys. 243 (1995)90, M.Dimitrijevic, L.Jonke, L.Moeller, E.Tsouchnika, J.Wess and M.Wohlgenannt, Czech.J.Phys. 54 (2004)1243; S.Doplicher, K.Fredenhagen and J.E.Roberts, Phys.Lett. B331 (1994) 39; S.Ghosh and P.Pal, Phys.Lett. B633 (2006)397 Phys.Lett.B 618 (2005)243 S.Ghosh, Phys.Lett.B 623 (2005)251

[7] G.Sundaram and Q.Niu, Phys.Rev.B B59(1999)14915; F.D.M.Haldane, Phys.Rev.Lett. 93 (2004) 206602 cond-mat/0408417 R.Shindou and K.-I Imura, Nucl. Phys. B720[FS](2005) 399 ; C.Zhang, A.M.Dudarev and Q.Niu, cond-mat/0507125

[8] P.Pureur et.al., cond-mat/0501482

[9] G.Tatara and H.Kawamura, J.Phys.Soc.Jpn 71 (2002) 2613;

[10] M.Onoda, G.Tatara and N.Nagaosa, J.Phys.Soc.Jpn. 73 (2002) 2624; H.Kawamura, Phys.Rev.Lett. 90 (2003) 047202, G.Tatara (correspondence).

[11] A. Berard, J. Lages and H. Mohrbach, Eur.Phys.J. C35 (2004) 373-381; S.Ghosh, Phys.Lett. B638 (2006)350

[12] L.Faddeev and R.Jackiw, Phys.Rev.Lett. 60 (1988) 1692.

[13] C.Duval et al., Mod.Phys.Lett B20 (2006) 373 ; Z.Horvath, P.A.Horvathy and L.Martina, cond-mat/0511099

[14] C. Duval and P. A. Horvthy, Phys.Lett. B, Volume 479, (2000) 284; V. P. Nair, Phys. Lett. B 505 (2001) 249 ; V. P. Nair and A. P. Polychronakos, Phys. Lett. B 505 (2001) 267.

[15] M.Chaichian, M.M.Sheikh-Jabbari and A.Tureanu, Phys. Rev. Lett. 86 (2001) 2716 ; M.Chaichian, A. Demichev, P.Presnajder, M.M.Sheikh-Jabbari and A.Tureanu, Nucl. Phys. B 611 (2001) 383.

[16] M.Chaichian, P.Presnajder, M.M.Sheikh-Jabbari and A.Tureanu, Phys.Lett. B527 (2002) 149; H. Falomir, J. Gamboa, M. Loeve, F. Mendez and J. C. Rojas, Phys. Rev. D 66 (2002) 045018.

[17] K.Li and S.Dulat, hep-th/0508193

[18] B. Mirza and M. Zarei, Eur. Phys. J. C 32 (2004) 583.

[19] T. Curtright, D. Fairlie and C. Zachos, Phys. Rev. D58 (1998) 25002; L. Mezincescu, hep-th/0007046 
[20] P.A.Horvathy, hep-th/0602133

[21] B.Nikolic, L.P.Zarbo and S.Welack, Phys.Rev. B72 (2005) 075335 . We thank D.Xiao for this.

[22] See for example, L. Schulman, Techniques and applications of path integrals, Wiley and Sons, N. Y. 1981; P.A.Horvathy, G. Morandi and E.C.G.Sudarshan, Il Nuovo Cimento D11, p. 201 (1989); H. Kleinert, Path Integrals in Quantum Mechanics, Statistics, and Polymer Physics, 2nd Ed. World Scientific (1995). 